\title{
Gauge fixing methods and Gribov copies effects in lattice QCD
}

\section{Paulo J. Silva* and Orlando Oliveira}

Centro de Física Computacional, Departamento de Física, Universidade de Coimbra, P-3004-516 Coimbra, Portugal

E-mail: psilva@teor.fis.uc.pt, orlando@teor.fis.uc.pt

We compare two Landau gauge fixing methods, aiming to find the global maximum of the gauge fixing functional. Moreover, a systematic effect of Gribov copies in the gluon and ghost propagators computed in Landau gauge is presented and discussed.

The XXV International Symposium on Lattice Field Theory

July 30 - August 42007

Regensburg, Germany

\footnotetext{
* Speaker.
} 


\section{Comparing Landau gauge fixing methods}

The formulation of QCD on the lattice does not require gauge fixing. However, for example, if one wishes to study the propagators of the fundamental fields, one must choose a given gauge. A possible choice is the so called Landau gauge, $\partial_{\mu} A_{\mu}=0$. On the lattice, the Landau gauge is implemented by searching for stationary points of

$$
F_{U}[g]=\sum_{x, \mu} \operatorname{Re}\left\{\operatorname{Tr}\left[g(x) U_{\mu}(x) g^{\dagger}(x+\hat{\mu})\right]\right\}
$$

over the gauge orbit of $U_{\mu}$. As any other local continuous gauge fixing condition [1,2], Landau gauge suffers from the so called Gribov copies problem [3], i.e. there are multiple solutions for the gauge condition in each gauge orbit. This rises the question on the possibility of a non-perturbative definition of the Landau gauge. A solution to this problem is to identify Landau gauge as the search of the unique [4], up to a global gauge transformation, absolute maxima of (1.1). Unfortunately, the search of a global maximum of a function on a multidimensional manifold is far from being trivial. For the particular case of the gauge under discussion, some methods have been devised which, hopefully, will be able to compute the absolute maximum of $F_{U}[g]$.

In this work we compare two of them, namely the CEASD method, described in [5], and the smeared gauge fixing, described in [6]. The CEASD method combines an evolutionary algorithm with the steepest descent (SD) method [7]. On the other hand, smeared gauge fixing relies on the smooting of the gauge fixing hypersurface by smearing the configuration.

We tested these two methods on $\mathrm{SU}(3) 16^{4}$ pure gauge Wilson action configurations ${ }^{1}$ for $\beta \in\{5.7,5.8,5.9,6.0,6.2\}$. For each $\beta$ value, five configurations were generated. For each configuration, $500 \mathrm{SD}$ gauge fixings were performed, starting from different randomly chosen points. This procedure gives an idea on the number of Gribov copies for each configuration, and defines a candidate for the global maximum of (1.1). As expected, the number of Gribov copies seems to increase with the physical volume of the lattice.

For each configuration, we have compared the global maximum obtained with the different 500 SD ('GMAX' in the figures) with the maxima computed by the CEASD method ('CEASD' in the figures), by smeared gauge fixing ('SMGF' in the figures) and by a single steepest descent applied to the configuration ('SD' in the figures).

The various plots in figure 1 show that the smeared gauge fixing only once was able to identify correctly the global maximum ( $\beta=5.9$ configuration $\mathrm{nr}$. 6000). On the other hand, the CEASD method was successful in all $\beta=6.0$ and $\beta=6.2$ configurations, and in 4 of the $\beta=5.9$ configurations. Curiously, the CEASD method found a larger $F_{U}$ than the one found by $500 \mathrm{SD}$ for one of the $\beta=5.8$ configurations. In what concerns the computation of the absolute maximum of $F_{U}[g]$, the CEASD method seems to be superior, but it is very computationally demanding. Indeed, it requires $\sim 30$ hours in a single Pentium IV, to be compared with $\sim 3$ hours for the smeared gauge fixing method or $\sim 30$ minutes for the steepest descent.

\footnotetext{
${ }^{1}$ All the configurations used in this work were generated with MILC code [14].
} 


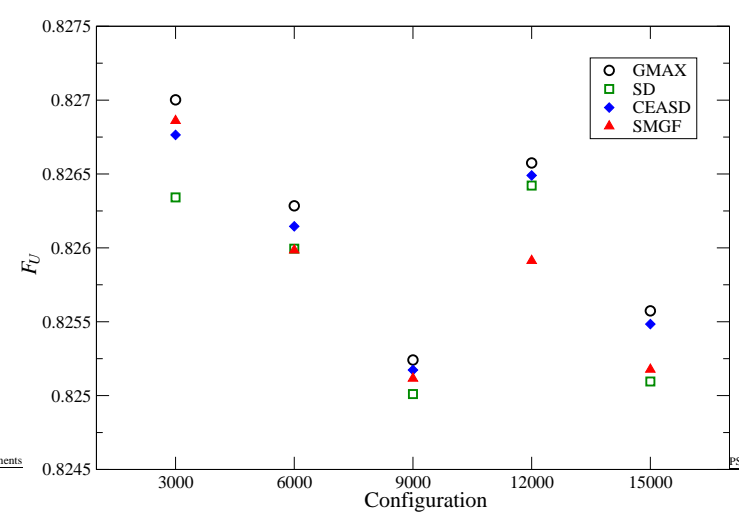

(a) $\beta=5.7$

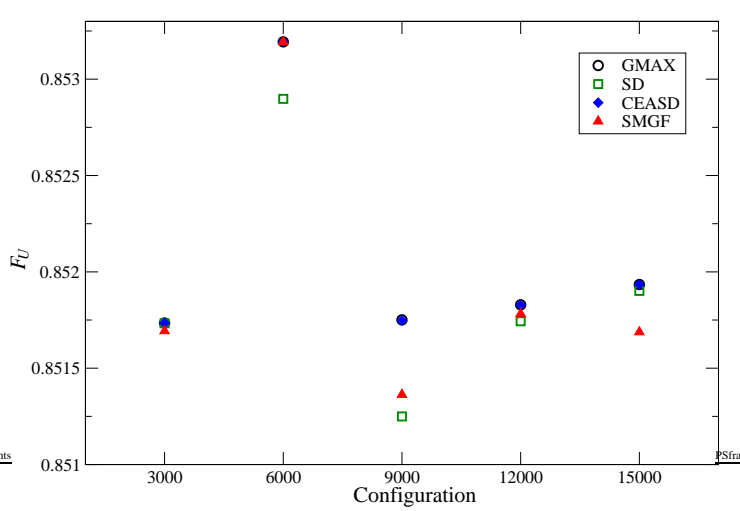

(c) $\beta=5.9$

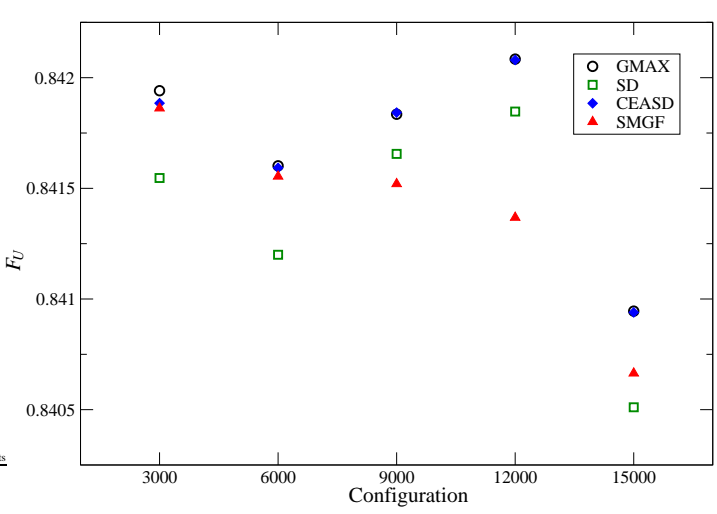

(b) $\beta=5.8$

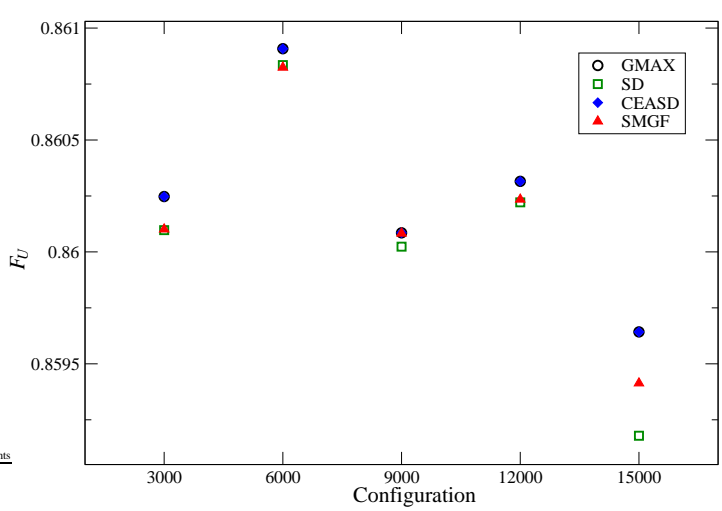

(d) $\beta=6.0$

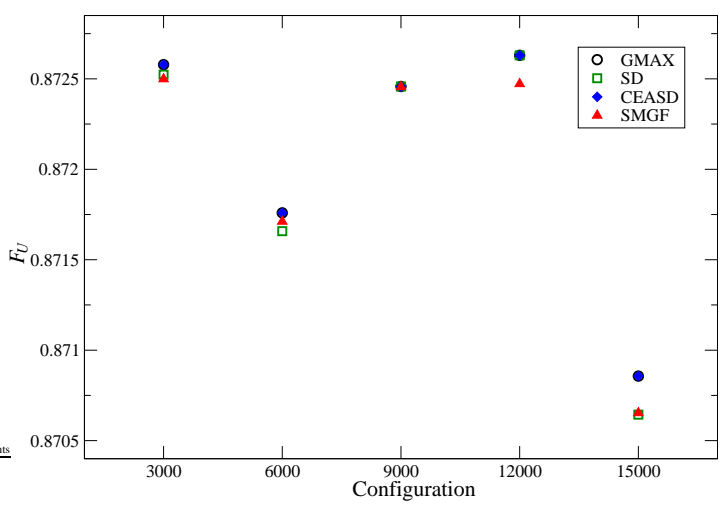

(e) $\beta=6.2$

Figure 1: $F_{U}$ maxima found by the different gauge fixing methods considered. 


\section{Gribov copies and the gluon and ghost propagators}

The investigation of the Gribov copies effects on the gluon and ghost propagators has been an active research field for some time - see for example, $[8,9,10,11]$ and references there in. Although in a recent work [12] it has been argued that the different maxima of $F_{U}$ do not change the infinite volume expectation values, this is not necessarily true on a finite lattice.

In this section, the effects of Gribov copies in the gluon [13] and ghost propagators are investigated using 302 configurations for a $12^{4}$ lattice with $\beta=5.8$. For each configuration, 500 SD, starting from different randomly chosen points, were performed and saved the gauge configurations associated to the largest maximum of $F_{U}$, the smallest maximum of $F_{U}$, and a few more random gauge configurations associated to intermediate values of $F_{U}$ maxima. Then, the propagators computed have been labelled by $\left\langle F_{U}\right\rangle$, the mean value of the corresponding $F_{U}$ associated to each gauge fixed configuration.

In this work, we compute the gluon propagator $D\left(q^{2}\right)$ using the following definitions (see [13] for more details)

$$
D\left(q^{2}\right)=\frac{2}{\left(N_{c}^{2}-1\right)\left(N_{d}-1\right) V} \sum_{\mu}\left\langle\operatorname{Tr}\left[A_{\mu}(\hat{q}) A_{\mu}(-\hat{q})\right]\right\rangle, \quad q \neq 0,
$$

and

$$
D\left(q^{2}=0\right)=\frac{2}{\left(N_{c}^{2}-1\right) N_{d} V} \sum_{\mu}\left\langle\operatorname{Tr}\left[A_{\mu}(\hat{q}) A_{\mu}(-\hat{q})\right]\right\rangle
$$

where

$$
A_{\mu}(\hat{q})=\sum_{x} \exp \left[-i \hat{q}\left(x+a \hat{e}_{\mu} / 2\right)\right] A_{\mu}\left(x+a \hat{e}_{\mu} / 2\right)
$$

and

$$
A_{\mu}\left(x+a \hat{e}_{\mu} / 2\right)=\frac{1}{2 i g_{0}}\left[U_{\mu}(x)-U_{\mu}^{\dagger}(x)\right]-\frac{1}{6 i g_{0}} \operatorname{Tr}\left[U_{\mu}(x)-U_{\mu}^{\dagger}(x)\right]+\mathscr{O}\left(a^{2}\right) .
$$

On the lattice, the discrete momenta available are given by

$$
\hat{q}_{\mu}=\frac{2 \pi n_{\mu}}{a L_{\mu}}, \quad n_{\mu}=0,1, \ldots L_{\mu} / 2,
$$

and

$$
q_{\mu}=\frac{2}{a} \sin \left(\frac{\hat{q}_{\mu} a}{2}\right) .
$$

The ghost propagator was computed using a plane wave source [8],

$$
G^{a b}(\hat{q})=\frac{1}{V}\left\langle\sum_{x, y}\left(M^{-1}\right)_{x y}^{a b} \exp [i \hat{q} \cdot(x-y)]\right\rangle
$$

and the ghost scalar function computed from

$$
G\left(q^{2}\right)=\frac{1}{N_{c}^{2}-1} \sum_{a} G^{a a}(\hat{q}) .
$$

In figure 2 we report the dependence of the gluon propagator in $\left\langle F_{U}\right\rangle$. The data shows that $D(0)$ increases as $\left\langle F_{U}\right\rangle$ increases. On the other hand, the low non-zero momenta $D\left(q^{2}\right)$ seem to be decreasing as $\left\langle F_{U}\right\rangle$ increases. The high momenta region seems to be blind to the choice of copy. 


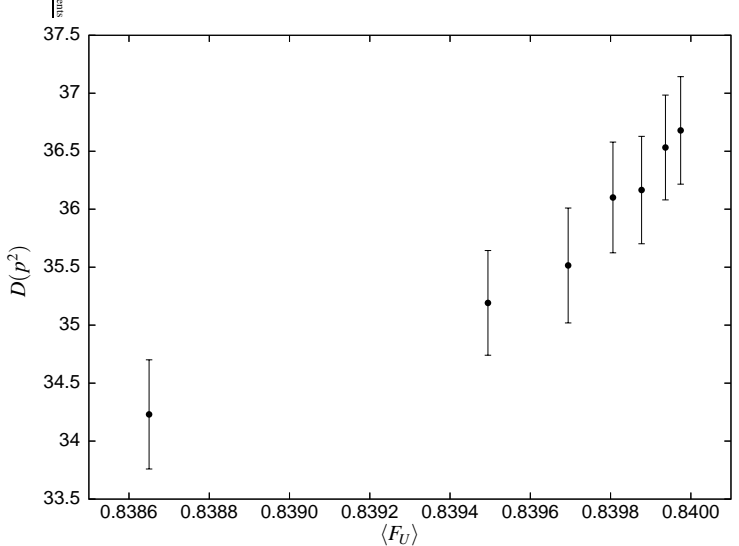

(a) $n_{\mu}=(0,0,0,0)$

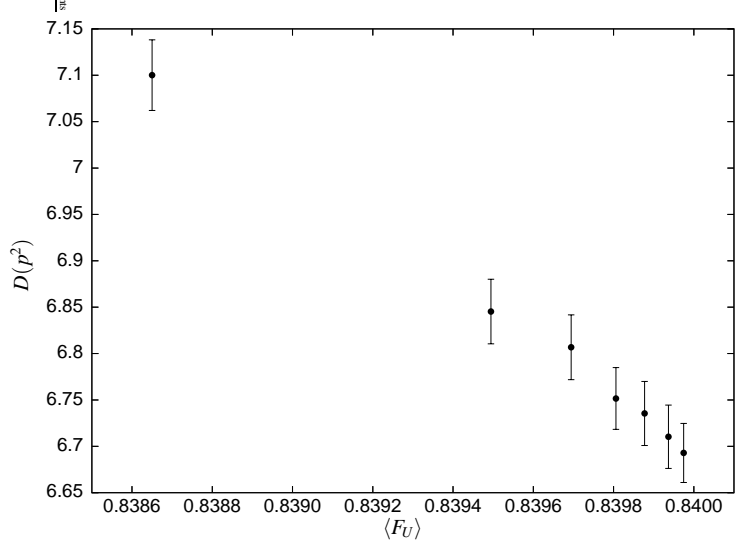

(c) $n_{\mu}=(1,1,0,0)$

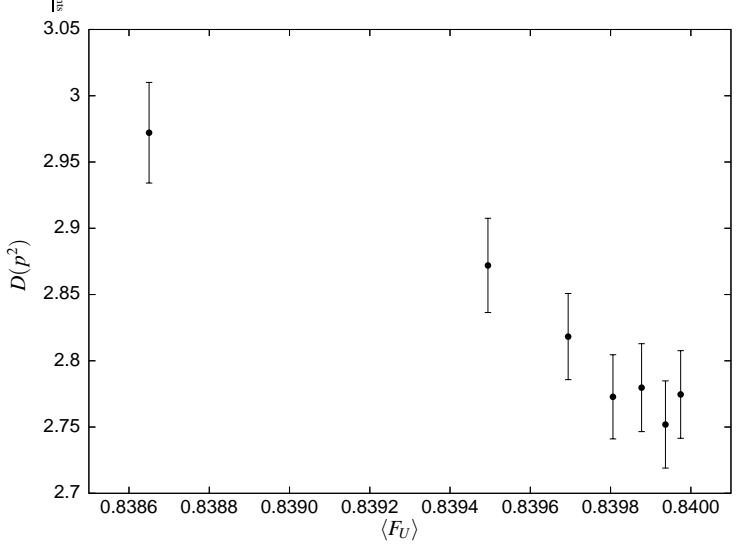

(e) $n_{\mu}=(1,1,1,1)$

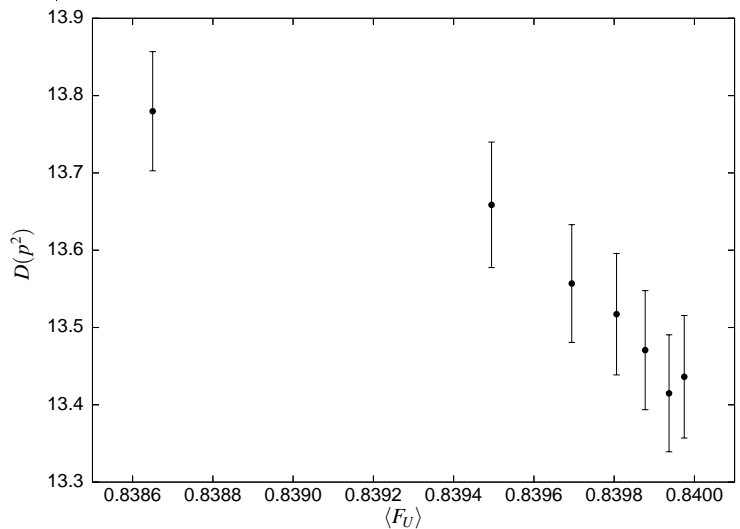

(b) $n_{\mu}=(1,0,0,0)$

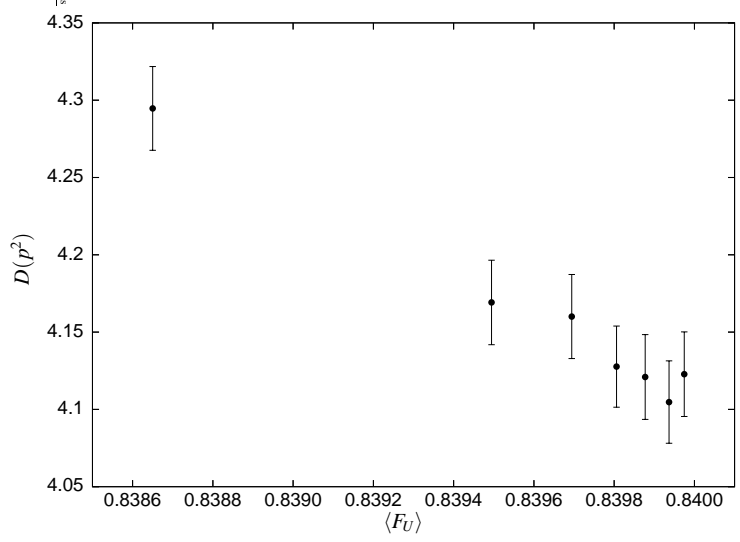

(d) $n_{\mu}=(1,1,1,0)$

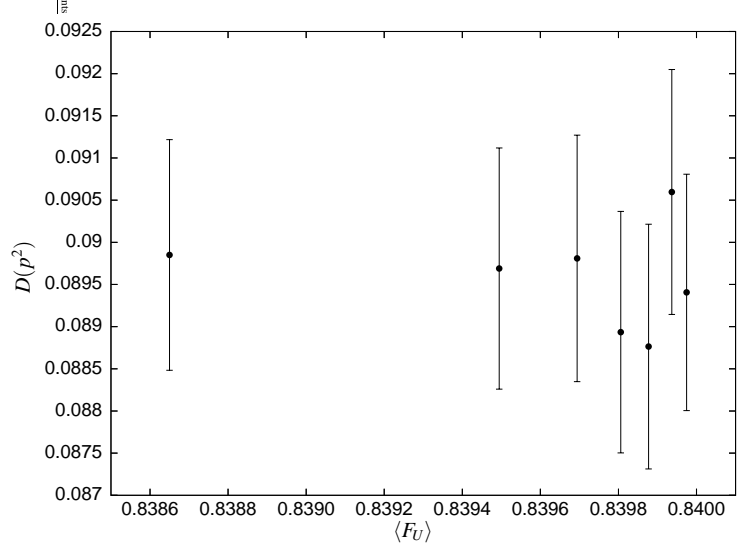

(f) $n_{\mu}=(6,6,6,6)$

Figure 2: Gluon propagator versus $\left\langle F_{U}\right\rangle$. 


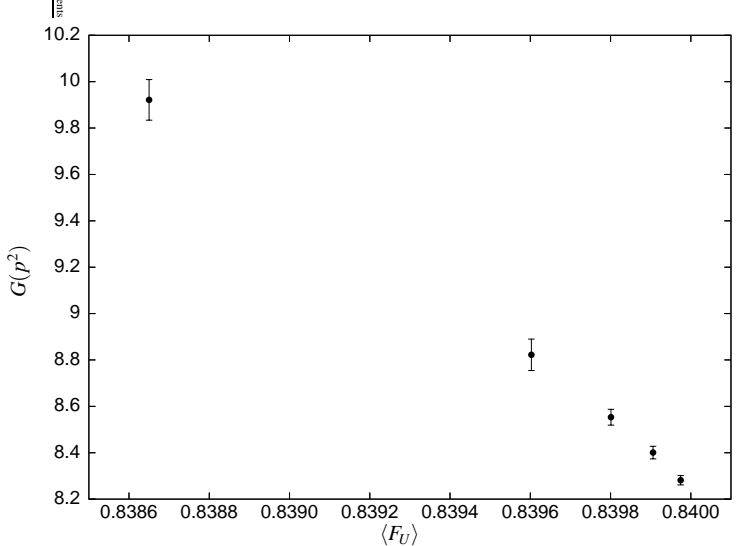

(a) $n_{\mu}=(1,0,0,0)$

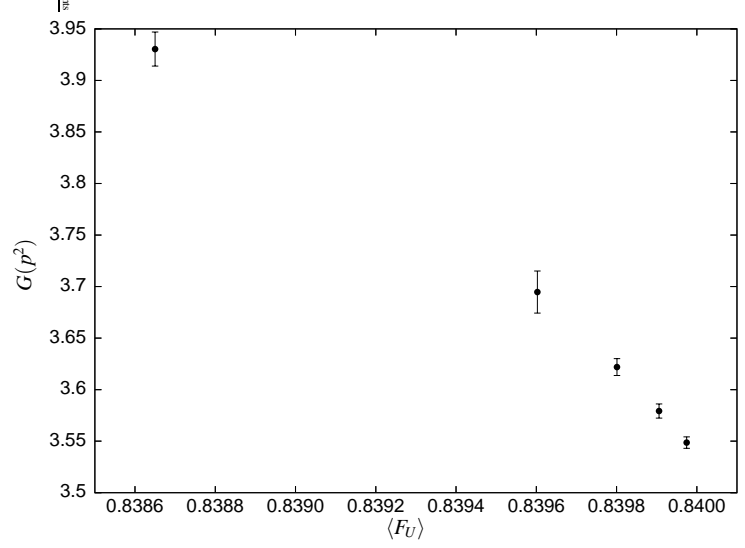

(c) $n_{\mu}=(1,1,0,0)$

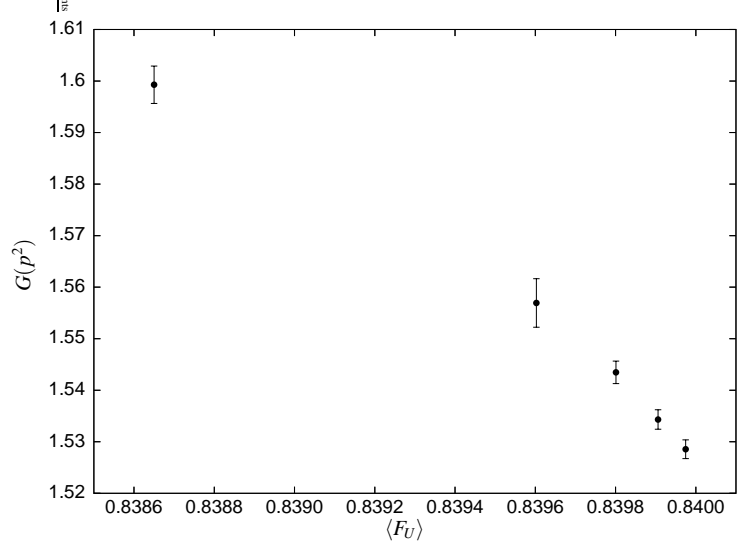

(e) $n_{\mu}=(1,1,1,1)$

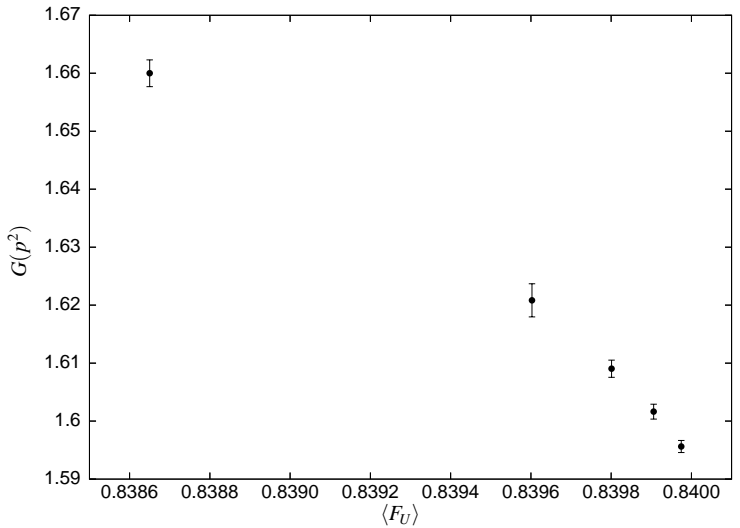

(b) $n_{\mu}=(2,0,0,0)$

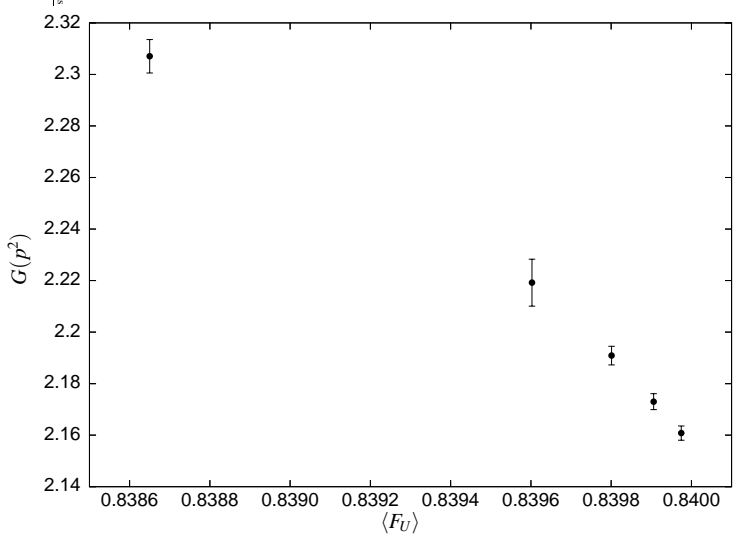

(d) $n_{\mu}=(1,1,1,0)$

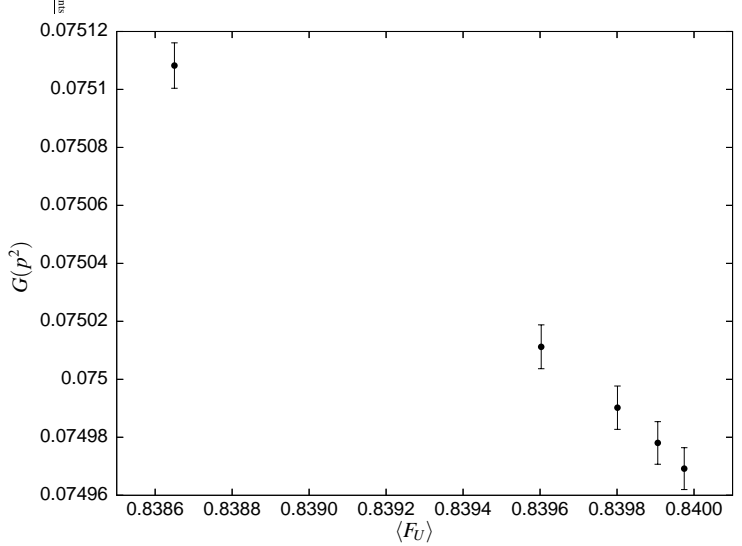

(f) $n_{\mu}=(6,6,6,6)$

Figure 3: Ghost propagator versus $\left\langle F_{U}\right\rangle$. 
These results show that Gribov copies are important only for the infrared regime. In what concerns the ghost propagator, see figure 3, their values decrease as $\left\langle F_{U}\right\rangle$ increases. Once more, the effect is bigger for low momenta (a $20 \%$ effect for the lowest momentum, reduced to $0.2 \%$ for the highest momentum considered).

\section{Conclusions}

For the lattice Landau gauge, we have compared two methods of gauge fixing tunned to approach the global maximum of $F_{U}[g]$. For a $16^{4}$ lattice, the CEASD method seems to perform better than smeared gauge fixing. Unfortunately, CEASD is quite computational demanding, not only in what concerns memory but also in CPU time.

In the second part of this paper, we investigated the effect of Gribov copies in the gluon and ghost propagators. Our data shows a clear dependence of gluon and ghost propagators on $\left\langle F_{U}\right\rangle$. Furthermore, the data shows that the changes on the propagators due to the choice of the maximum of $F_{U}[g]$ are mainly on the infrared region.

\section{Acknowledgments}

This work was supported by FCT via grant SFRH/BD/10740/2002, and partially supported by projects POCI/FP/63436/2005 and POCI/FP/63923/2005.

\section{References}

[1] I. M. Singer, Comm. Math. Phys. 60 (1978) 7.

[2] T. P. Killingback, Phys. Lett. B138 (1983) 87.

[3] V. N. Gribov, Nucl. Phys. B139 (1978) 1.

[4] G. Dell'Antonio, D. Zwanziger, Comm. Math. Phys. 138 (1991) 291.

[5] O. Oliveira, P. J. Silva, Comp. Phys. Comm. 158 (2004) 73, arXiv: hep-lat/0 0309184.

[6] J. E. Hetrick, Ph. de Forcrand, Nucl.Phys. B (Proc.Suppl.) 63 (1998) 838, arXiv:hep-lat/9710003.

[7] C. H. T. Davies, G. G. Batrouni, G. P. Katz, A. S. Kronfeld, G. P. Lepage, P. Rossi, B. Svetitsky and K. G. Wilson, Phys. Rev. D37 (1988) 1581.

[8] A. Cucchieri, Nucl. Phys. B508 (1997) 353, arXiv : hep-lat/ 9705005.

[9] A. Sternbeck, E.-M. Ilgenfritz, M. Müller-Preussker, A. Schiller, Phys. Rev. D72 (2005) 014507, arXiv:hep-lat/0506007.

[10] I. L. Bogolubsky, G. Burgio, M. Müller-Preussker, V. K. Mitrjushkin, Phys. Rev. D74 (2006) 034503, arXiv:hep-lat/0511056.

[11] I. L. Bogolubsky, V. G. Bornyakov, G. Burgio, E. M. Ilgenfritz, M. Müller-Preussker, V. K. Mitrjushkin, arXiv:0707.3611 [hep-lat].

[12] D. Zwanziger, Phys. Rev. D69 (2004) 016002, arXiv : hep-ph / 0303028.

[13] P. J. Silva, O. Oliveira, Nucl. Phys. B 690 (2004) 177, arXiv: hep-lat / 0403026.

[14] http://www.physics.indiana.edu/ sg/milc.html. 